\title{
Effects of porous walled tubes on detonation transmission into unconfined space
}

\author{
Navid Mehrjoo ${ }^{1}$, Yuan Gao², Charles Basenga Kiyanda ${ }^{1 \dagger}$, Hoi Dick Ng${ }^{1}$ and John H.S. Lee \\ ${ }^{1}$ Concordia University \\ Department of Mechanical and Industrial Engineering \\ Montréal, H3G 1M8, Canada \\ ${ }^{2}$ McGill University \\ Department of Mechanical Engineering, Montréal, H3A 2K6, Canada \\ ${ }^{\dagger}$ Corresponding Author \\ Department of Mechanical and Industrial Engineering \\ Concordia University \\ 1455 de Maisonneuve Blvd. West \\ Montréal, H3G 1M8, Canada \\ e-mail: kiyanda@encs.concordia.ca \\ Tel.: (514) 848-2424 (ext. 7121)
}

Fax: (514) 848-3175

Manuscript submitted to the $35^{\text {th }}$ Symposium (International) on Combustion

Colloquium: Detonations, Explosions and Supersonic Combustion

Preferred type of presentation: oral

Total length of paper (method 1): 5831

main text: 3083

references: 315

figure 1 with captions: 315

figure 2 with captions: 260

figure 3 with captions: 468

figure 4 with captions: 415

figure 5 with captions: 325

figure 6 with captions: 325

figure 7 with captions: 325 


\begin{abstract}
Experiments were carried out to investigate the failure mechanisms in the critical tube diameter phenomenon for stable and unstable mixtures. It was previously postulated that in unstable mixtures where the detonation structure is highly irregular, the failure during the diffraction is caused by the suppression of the instability responsible for the generation of local explosion centers. In stable mixtures, typically with high argon dilution and where the detonation is characterized by very regular cell, the failure is driven by the excessive global front curvature above which a detonation cannot propagate. To discern these two failure mechanisms, porous wall tubes are used to attenuate the transverse instability before the detonation emerges into the unconfined space. Porous sections with length $L / D$ from 0 to 3.0 are used with two confined tube diameters $D=12.7$ and $15.5 \mathrm{~mm}$. The present results show that when porous wall tubes are used, the critical pressure for unstable $\mathrm{C}_{2} \mathrm{H}_{2}+2.5 \mathrm{O}_{2}$ and $\mathrm{C}_{2} \mathrm{H}_{2}+5 \mathrm{~N}_{2} \mathrm{O}$ mixtures increases significantly. In contrast, for stable argon diluted $\mathrm{C}_{2} \mathrm{H}_{2}+2.5 \mathrm{O}_{2}+70 \%$ mixtures, the results with porous wall tubes exhibit little variation up to $L / D=2.5$. For $L / D>2.5$ a noticeable increase in critical pressure for argon diluted mixtures is also observed. This is dominantly caused by the slow mass divergence through the porous material inducing a curvature on the detonation front even before it emerges into the open area. The present experiment again demonstrates the importance of the transverse wave instability for typical hydrocarbon mixtures in critical situations such as the critical tube diameter experiment. For special cases such as highly argon diluted mixtures, the instability does not play a significant role in the failure and the propagation is controlled dominantly by the global curvature effect and the shock-ignition mechanism.
\end{abstract}

Keywords: Detonation; Critical tube diameter; Porous walled tube; Instability; Failure mechanism 


\section{Introduction}

The critical tube diameter phenomenon has long been a classical problem in detonation research. It not only provides a well-defined fundamental problem in understanding both initiation and failure of detonation waves, but knowledge of the critical tube diameter also has practical applications such as in the design of initiators for pulse detonation engines, e.g., when the detonation transmits from the small pre-detonator to the main thrust tube of the pulse detonation engine [1-3]. The critical tube diameter, $D_{c}$, is defined as the minimum diameter of a round tube for which a detonation emerging from it to an open space can continue to propagate. The critical tube diameter can be considered as an alternative length scale that provides an assessment of the relative detonation sensitivity of combustible mixtures [4]. This scale is in contrast to the detonation cell width which, while a fundamental length scale of the detonation structure, can present significant variability when measured. We are thus moving from a measurement with high statistical variability (cell size) [5] to a more reproducible measurement (critical diameter).

As the detonation diffracts during the transmission from the confined tube to the open area, expansion waves from the periphery will influence the propagation of the diverging detonation, leading to failure. To describe the effect of these expansion waves on the propagation of the detonation during diffraction, Lee [6] suggested that the failure mechanism is governed by the detonation instability of the given explosive mixtures. Two modes of failure were proposed: one is a local failure mechanism that is linked to the dynamics of instabilities in undiluted mixtures, while the other mechanism supposes failure

is due to the excessive curvature of the global front, in stable mixtures highly diluted with argon. For common hydrocarbon mixtures in which detonations are unstable with highly irregular cellular structures, open-shutter photographs $[6,7]$ reveal that successful transmission is often found to originate from a localized region in the failure wave, which eventually amplifies to sustain the detonation front propagation in the open area. Hence, failure is linked to the suppression of perturbations, inherent instabilities that otherwise generate new, localized explosion centers. On the other hand, for stable 
detonations with very regular cells, typical in highly argon diluted mixtures [8], failure is predominantly caused by excessive curvature of the entire detonation front, whereby the corner expansion waves distribute the curvature over the detonation surface.

To discern these two modes of failure responsible for the critical tube diameter phenomenon, a simple experiment was recently performed by Mehrjoo et al. [9]. Unlike most previous studies on transmission promotion using techniques of various orifice shapes with relatively large blockage or distribution of transmission points (see [7] and references therein), Mehrjoo et al. [9] investigates the effect of a finite perturbation generated by placing a small slender needle that serves as an obstacle with a small blockage ratio at the tube exit diameter just before the detonation diffraction and observing the phenomenon's response. In this previous study, it is found that the small perturbation can have an effect in undiluted hydrocarbon mixtures resulting in the decrease of the critical pressure for successful detonation transmission. In other words, the disturbance caused by the small obstacle promotes transmission and this result shows that local hydrodynamic instabilities are significant for detonation diffraction in typical, undiluted, unstable mixtures considered for detonation-based propulsion systems. For mixtures such as highly diluted argon mixtures, which are stable with regular cellular patterns, the results using this small needle perturbation do not show a significant difference between the perturbed and unperturbed cases. This is explained by the fact that the effect of the small perturbations on the global curvature for the emergent detonation wave is minimal.

This present study proposes another simple experiment to illustrate the effect of instability on the detonation transmission from a confined tube to an open space by investigating the suppression of perturbations rather than their generation. Unlike the experiment using slender obstacles to generate perturbations, it is possible to suppress “instabilities”, i.e. “transverse waves”, inside the confined tube before the detonation wave emerges into the open area. This can be done by using acoustically absorbent material, which has the ability to attenuate the transverse waves associated with cellular detonation fronts. Such a method using acoustic absorption was indeed employed by Dupré et al. [10], Teodorczyk 
\& Lee [11] and more recently by Radulescu \& Lee [12] to demonstrate the essential role of transverse waves on the propagation of detonation waves in circular tubes or thin channels. In this work, we extend results from these earlier studies onto the critical tube diameter problem and consider the effect of absorbing walls placed at the exit of the confined tube before the detonation emerges into the open area. This experiment illustrates the effect of transverse waves on the detonation transmission, again confirms the two postulated failure mechanisms, and also contributes to practical applications such as in the design of detonation arrestors, a device to quench or stop detonation propagation from one confined region to another, larger space. While previous studies have sought to link the detonation cell size to the critical tube diameter, we concentrate here solely on the response of different mixtures, representative of regular and irregular mixtures, to changes in the porosity of the side wall in the critical tube diameter experiment. The detonation cell size is visualized only to ascertain that the chosen porous material has an effect on the transverse wave activity, but the magnitude of that effect is not quantified.

\section{Experimental setup}

The experiments were carried out in a high-pressure, spherical explosion chamber. The chamber is $20.3 \mathrm{~cm}$ in diameter with a $5.1 \mathrm{~cm}$ thick wall. The chamber's body is connected, at the top, to a $41.8 \mathrm{~cm}$ long vertical, round tube that can be fitted with porous walls. Two different inner diameters, $D=$ $15.5 \mathrm{~mm}$ and $12.7 \mathrm{~mm}$, were considered for each type of combustible mixture. Figure 1a shows the schematic of the experimental setup.

For the present study, the exit of the vertical tube was made porous using $1 / 2$ ” inner diameter soaker hose (Colorite SNUER12025 cut to fit the tube inner diameter) made of extruded rubber material. The length of the porous wall was varied from $L=0$ to $3 D$ where $L / D=0$ means no porous material was inserted. The inner tube diameter was kept constant whether or not the porous insert was present as shown in Fig. 1b. The effect of the porous material mounted on the wall was also tested in a detonation 
tube facility shown in Fig. 2. It consists of a steel driver section $65 \mathrm{~mm}$ in diameter and $1.3 \mathrm{~m}$ long. A polycarbonate test tube of various diameters was attached to the end of the driver tube and the porous material was mounted in the middle of the test tube. The smoked foil technique was used to reveal the attenuation effect of this porous wall section.

Stoichiometric mixtures of acetylene/nitrous oxide, acetylene/oxygen, and acetylene/oxygen with $70 \%$ argon dilution were tested in this experimental study. The first two mixtures exhibit irregular (unstable) cellular detonation structures, while the latter exhibits a stable detonation front with regular cellular patterns $[8,13]$. These mixtures were prepared by the method of partial pressures in separate gas bottles and were allowed to mix in the bottle for at least 24 hrs in order to ensure mixture homogeneity. For each experiment, the experimental setup was initially evacuated to approximately $100 \mathrm{~Pa}$ and then filled through the valve with mixtures at various initial test pressures $p_{0}$ by which the mixture sensitivity was varied. The initial pressure was monitored by an Omega pressure transducer (0-30 psi) with an accuracy of $\pm 0.25 \%$ full scale. The lower pressure range was also checked with a more accurate digital manometer model HHP242-015A (0 to 15 psi) with an accuracy of $\pm 0.10 \%$ full scale. A high-voltage spark ignition source was used to directly initiate a Chapman-Jouguet (CJ) detonation that then propagated through the vertical round tube to finally emerge into the large spherical chamber [14].

The procedure used to determine whether the emerging detonation from the confined tube was successfully transmitted into the open space is the same as described in [9, 15-16]. A piezoelectric pin (CA-1136, Dynasen Inc.) is located at the bottom wall of the chamber and measures the time-of-arrival of the wave. The time of arrival of successful/unsuccessful detonation transmission measured by the piezoelectric shock pin was compared to the time of ignition in the vertical tube and, hence, the wave velocity was deduced. Using these time-of-arrival (TOA) measurements, it was possible to distinguish between successful and failed detonation transmission from the significant difference in the time scale (or velocity) of the phenomenon. For successful transmission, the wave velocity typically obtained was around 90\% CJ detonation velocity. For an unsuccessful transmission, the velocity of the diverging 
wave computed using the TOA signal from the shock pin was typically below $25 \%$ of the CJ velocity value, e.g., see [15]. For some experiments, a photo probe located at the top of the spherical bomb (i.e., near the end of the vertical tube) was also used to record the time of arrival of the wave and check whether a successful detonation was first initiated in the vertical tube from the computed wave velocity. The critical condition for each mixture was characterized by the critical pressure below which the detonation fails to emerge into the large spherical chamber.

\section{Results and discussion}

The smoked foil technique was used to look at the influence of the porous wall on the cellular detonation structure. As an example, Fig. 3 shows the smoked foils obtained for stoichiometric $\mathrm{C}_{2} \mathrm{H}_{2}+2.5 \mathrm{O}_{2}$ at different initial pressures. At an initial pressure sufficiently high for the detonation front to be multicellular, the smoked foils indicate, for all mixtures, a cell size increase and a subsequent return to the original cellular pattern after passage of the wave through the porous section. The effect of attenuation by the porous wall can be seen more clearly when the initial pressure is reduced. The damping by the porous media then causes the detonation wave to change from a multi-headed cellular front to a single headed spin downstream of the porous medium. Far away from the perturbation, the detonation reestablishes itself back to a multi-cellular front. For the case of $p_{o}=2 \mathrm{kPa}$, the incident detonation fails completely after passing through the porous wall section. Similar effects are observed for the stable mixtures with high argon dilution (i.e., stoichiometric $\mathrm{C}_{2} \mathrm{H}_{2}+2.5 \mathrm{O}_{2}+70 \% \mathrm{Ar}$ ) as shown in Fig. 4. The

results from this experiment thus indicate that the porous material used has the ability to damp out some transverse waves at the front for both stable and unstable mixtures.

Critical tube diameter experiments were then carried out with the porous material inserted on the tube wall, close to the exit of the tube. For each combustible mixture and porous media aspect ratio $L / D$, detonation transmission measurements were performed at different initial pressures to vary the 
sensitivity of the mixture. As in previous work, each experiment was repeated 8 times for each mixture and initial condition to ensure statistical convergence and reproducibility of the results, as well as to identify accurately the critical pressure value above which successful detonation transmission can occur. The critical pressure is defined by the upper limit boundary above which at least $75 \%$ of the tests at the same initial condition give a successful transmission of the detonation wave into the open space [9]. An example of the raw measurement data is given in Fig. 5, showing the Go/No-go plot (or successful/unsuccessful transmission) as a function of initial pressure with $L / D=0$ (non-porous) and $L / D=1$. In these plots, the overlap between the symbols representing successful $(\mathrm{O})$ and unsuccessful (X) transmission indicates that there is a mixed result among the 8 experimental shots repeated at that particular initial pressure. Such occurrence near critical conditions can be due to inherent sources of experimental variability and is typical for any detonation experiment. In this study of critical tube diameter, the range of uncertainty is not as significant as that of measurements of critical energy for direct initiation or detonation cell size.

Using the data as given in Fig. 5, it is possible to identify the critical pressure limit below which the detonation fails to transmit into the open area. Figures 6 and 7 show the results for the two diameters in all three tested combustible mixtures. For the unstable mixtures, stoichiometric $\mathrm{C}_{2} \mathrm{H}_{2}+2.5 \mathrm{O}_{2}$ and $\mathrm{C}_{2} \mathrm{H}_{2}$ $+5 \mathrm{~N}_{2} \mathrm{O}$, the results indicate that the porous wall has a significant effect on the critical tube diameter phenomenon. Even with $L / D=0.50$, there is already a significant increase in the critical pressure.

For the stoichiometric $\mathrm{C}_{2} \mathrm{H}_{2}+2.5 \mathrm{O}_{2}$ with $L / D=0.50$, the critical pressure increases from $17 \mathrm{kPa}$ to $21 \mathrm{kPa}$ and $11 \mathrm{kPa}$ to $17 \mathrm{kPa}$ for $D=12.7 \mathrm{~mm}$ and $D=15.5 \mathrm{~mm}$, respectively (or equivalently a difference of about $19 \%$ and 35\% where \% difference is defined by $[100 \%-(x / y \cdot 100 \%)]$ with $x$ and $y$ denoting the lower and higher number). A similar increase is also observed for the $\mathrm{C}_{2} \mathrm{H}_{2}+5 \mathrm{~N}_{2} \mathrm{O}$ mixtures with even the lowest $L / D=0.50$, respectively 8 and $9 \mathrm{kPa}$ increase for $D=12.7 \mathrm{~mm}$ and $D=$ $15.5 \mathrm{~mm}$ (or a difference of 8.7\% and 12\%). For these two mixtures, Fig. 6 also indicates that increasing 
the length of the porous walled section causes an exponential increase in the critical pressure limit and eventually no transmission can be observed within the allowable initial pressure for the experiment.

In contrast, the result for the diluted $\mathrm{C}_{2} \mathrm{H}_{2}+\mathrm{O}_{2}+70 \% \mathrm{Ar}$ mixtures shows little dependence on the presence of the porous wall section in the tube. For $L / D$ up to 2.5 , the critical pressure limit remains essentially constant (within 1-2 kPa). In other words, the critical condition for successful detonation transmission is not very sensitive to the transverse wave attenuation by the porous media and the flow instability has no major effect on this dynamic parameter of detonation for the diluted mixtures where the detonation wave structure is highly regular. However, for the largest $L / D=3.0$ used in this work there is an increase in the critical pressure limit. The dominant mechanism may not be caused by the transverse wave attenuation. This critical pressure increase is likely due to the excessive mass divergence into the porous wall [12], leading to the slow distribution of frontal curvature, for long enough $L / D$, even before the wave emerges into the open area.

In critical situations where the detonation propagation is prompt to failure, the instability at the cellular front can play an important role on the dynamics of the detonation wave. For unstable mixtures with highly irregular cellular detonation front, it is postulated that the detonation propagation or transmission into the open area relies on the instabilities at the front, i.e., the transverse waves [6]. The present experiment provides support of this conjecture. For the undiluted $\mathrm{C}_{2} \mathrm{H}_{2}+2.5 \mathrm{O}_{2}$ and $\mathrm{C}_{2} \mathrm{H}_{2}+5$ $\mathrm{N}_{2} \mathrm{O}$ mixtures, considered unstable, the attenuation caused by the porous media suppressed the front perturbations and the wave, during the diffraction process, fails to re-generate local explosion centers necessary for a successful transmission. In contrast, for the argon diluted mixture, more stable and exhibiting a very regular detonation front, the instabilities do not play a prominent role and the failure mechanism is proposed to be dominantly caused by the global curvature. The present results are in good agreement with this line of thought. Unlike the cases with unstable mixtures, a short to moderately long porous wall section is shown to have no immediate effect on the critical tube diameter phenomenon. This is due to the fact that the transverse wave attenuation, once the planar detonation enters the porous 
wall section and before it emerges into the open, unconfined area, does not modify immediately the global front curvature of the detonation wave. Hence, the critical condition for transmission with and without a porous wall with $L / D<2.5$ shows little variation. The effect of the porous wall may become significant if its length is large enough to allow the development of frontal curvature due to mass divergence, while the wave is still propagating in the porous walled section, before it emerges into the open area.

\section{Conclusion}

This work is an experimental study of detonation dynamics aimed at understanding the instability of the front that results in different failure mechanisms in the critical tube diameter phenomenon. Experiments using porous walled tubes were carried out to investigate how a self-sustained detonation propagating in a confined tube transmits into an open space, and to confirm the two postulated mechanism governing the successful transmission or failure in the two different types of mixtures (one with highly regular cellular pattern and the other with highly unstable detonation front).

Using a porous wall section near the exit of the confined tube to attenuate the transverse waves, the effect of instability on the failure mechanism of detonation wave diffraction is illustrated for typical hydrocarbon unstable mixtures. From the present, simple experiment, results demonstrate that for unstable mixtures, the successful transmission relies heavily on the frontal instability to generate local explosion centers. Suppression of instability by the porous media before the detonation emerges into the open space causes a significant increase in critical pressure limit for successful transmission. These results thus confirm the failure mechanism consisting in the suppression of instabilities. For stable mixtures such as those highly diluted with argon, the transverse waves at the front are typically weak and the immediate attenuation of these waves by the porous media does not significantly affect the

critical conditions for detonation transmission. The failure during the diffraction is therefore caused by 
the excessive global curvature above which a detonation cannot maintain its self-sustained propagation. This is also found in the present experiments where for long enough damping sections, the mass divergence through the porous wall has sufficient time to distribute its effect and generate front curvature before the detonation wave emerges into the open space, possibly eventually causing failure inside the porous walled section. The already curved detonation therefore will lead to a different critical condition, i.e., an increase of the critical pressure limit for $L / D>2.5$.

\section{Acknowledgments}

This work is supported by the Natural Sciences and Engineering Research Council of Canada (NSERC). 


\section{References}

[1] G.D. Roy, S.M. Frolov, A.A. Borisov, D.W. Netzer, Prog. Energy Combust. Sci. 30 (2004) 545672.

[2] C.P. Li, K. Kailasanath, Proc. Combust. Inst., 28 (2000) 603-609.

[3] H.Y. Fan, F.K. Lu, Proc. Inst. Mech. Eng. Part G: J. Aero. Eng. 222 (5) (2008) 673-686.

[4] J.H. Lee, Annu. Rev. Fluid Mech. 16 (1984) 311-336.

[5] J.E. Shepherd, I.O. Moen, S.B. Murray, P.A. Thibault, Proc. Combust. Inst. 21 (1986) 1649-1658.

[6] J.H.S. Lee, in: J. Bowen (Ed.), Dynamics of Exothermicity, Gordon and Breach, Amsterdam, 1996, pp. 321.

[7] A.A. Vasil'ev, in: F. Zhang, (Ed.), Shock Waves Science and Technology Library, Vol 6: Detonation Dynamics, Springer, Berlin Heidelberg (2012) Chap. 4.

[8] M.I. Radulescu, H.D. Ng, J.H.S. Lee, B. Varatharajan, Proc. Combust. Inst. 29 (2002) 2825-2831.

[9] N. Mehrjoo, B. Zhang, R. Portaro, H.D. Ng, J.H.S. Lee, Shock Waves 24 (2014) 219-229.

[10] G. Dupré, O. Peraldi, J.H.S. Lee, R. Knystautas, Prog. Astro. Aero. 114 (1988) 248-263.

[11] A. Teodorczyk, J.H.S. Lee, Shock Waves 4 (1995) 225-236.

[12] M.I. Radulescu, J.H.S. Lee, Combust. Flame 131 (1-2) (2002) 29-46.

[13] H.D. Ng, F. Zhang, in: F. Zhang, (Ed.), Shock Wave Science and Technology Reference Library, Vol. 6, Springer, Berlin Heidelberg (2012) Chap. 3.

[14] V. Kamenskihs, H.D. Ng, J.H.S. Lee, Combust. Flame 157(9) (2010) 1795-1799.

[15] B. Zhang, H.D. Ng, J.H.S. Lee, J. Loss Prev. Proc. Ind. 26 (2013) 1293-1299.

[16] B. Zhang, H.D. Ng, J.H.S. Lee, Combust. Flame 159 (9) (2012) 2944-2953. 


\section{Figure Captions}

Fig. 1. Schematic of a) the experimental facility; and b) porous walled tube.

Fig. 2. Porous walled region inside the test section of the detonation tube facility.

Fig. 3. Smoked foil measurement showing the cellular structure of the detonation before and after the passage of the porous walled tube in stoichiometric $\mathrm{C}_{2} \mathrm{H}_{2}+2.5 \mathrm{O}_{2}$ mixtures at different initial pressures.

Fig. 4. Smoked foil measurement showing the cellular structure of the detonation before and after the passage of the porous walled tube in stoichiometric $\mathrm{C}_{2} \mathrm{H}_{2}+2.5 \mathrm{O}_{2}+70 \%$ Ar mixtures at different initial pressures.

Fig. 5. Go/No-go plots as a function of initial pressure for the three combustible mixtures.

Fig. 6. The effect of porous walls on the critical pressure for successful detonation transmission for a) $D$ $=12.7 \mathrm{~mm}$; and b) $D=15.5 \mathrm{~mm}$ in two unstable stoichiometric $\mathrm{C}_{2} \mathrm{H}_{2}+2.5 \mathrm{O}_{2}$ and $\mathrm{C}_{2} \mathrm{H}_{2}+5 \mathrm{~N}_{2} \mathrm{O}$ mixtures.

Fig. 7. The effect of porous walls on the critical pressure for successful detonation transmission for a) $D$ $=12.7 \mathrm{~mm}$; and b) $D=15.5 \mathrm{~mm}$ in stable stoichiometric $\mathrm{C}_{2} \mathrm{H}_{2}+2.5 \mathrm{O}_{2}+70 \% \mathrm{Ar}$ mixtures. 


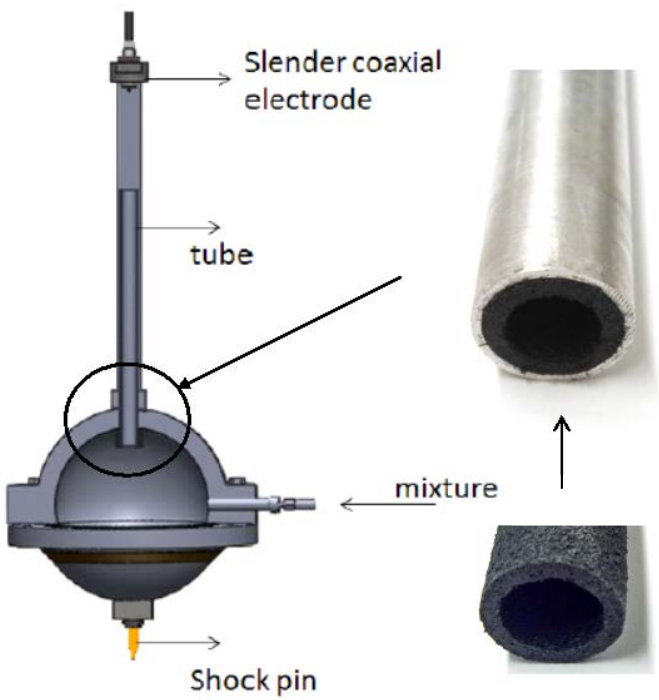

(a)
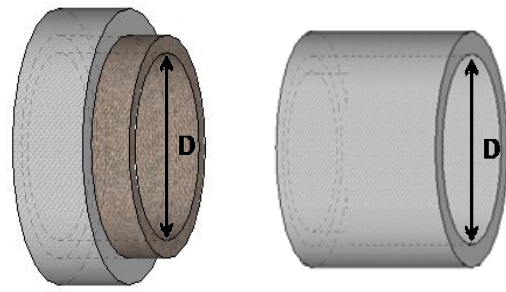

(b)

Fig. 1 

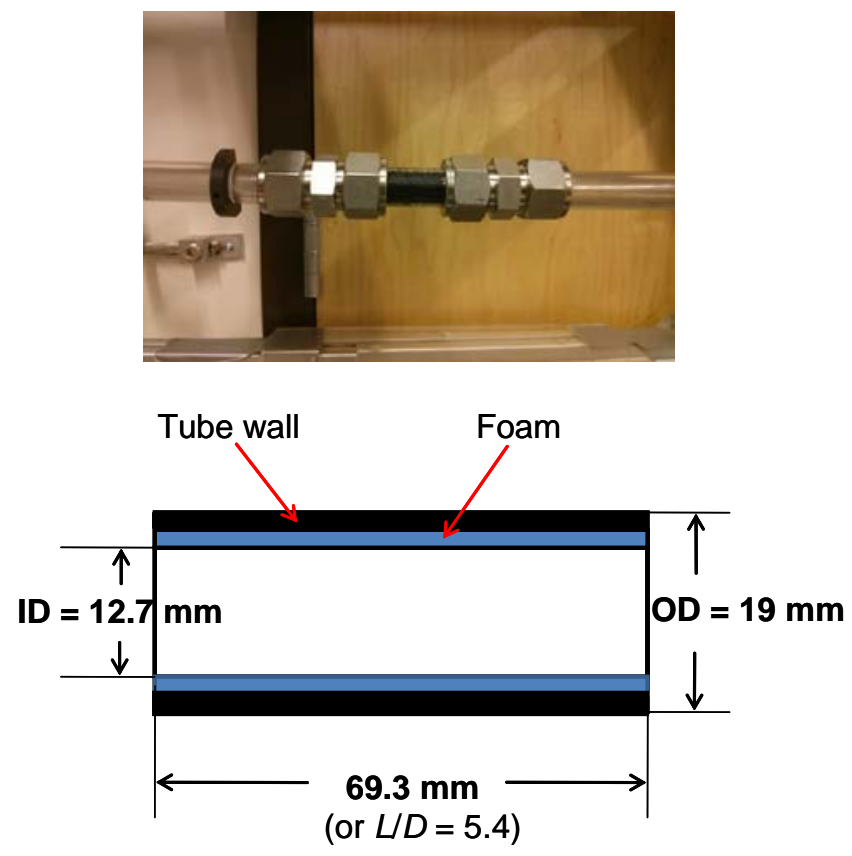

Fig. 2

Page 15 of 20 


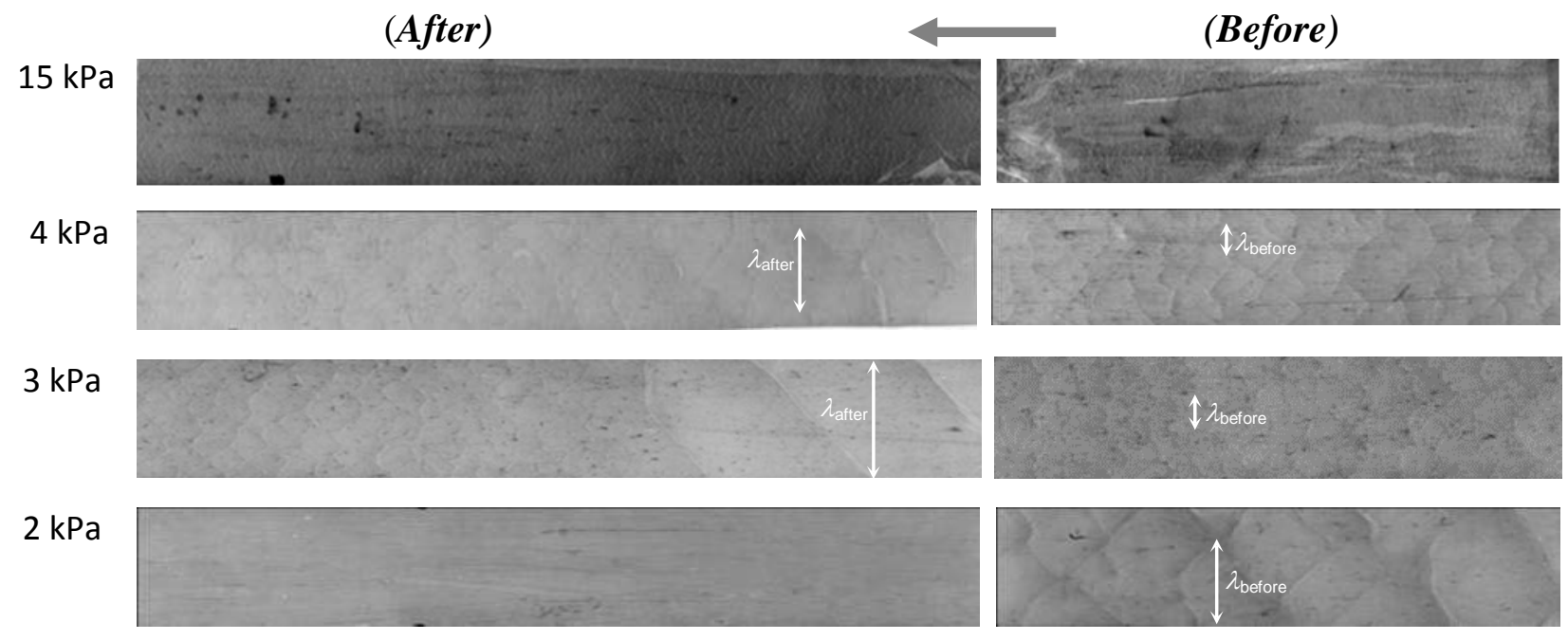

Fig. 3 


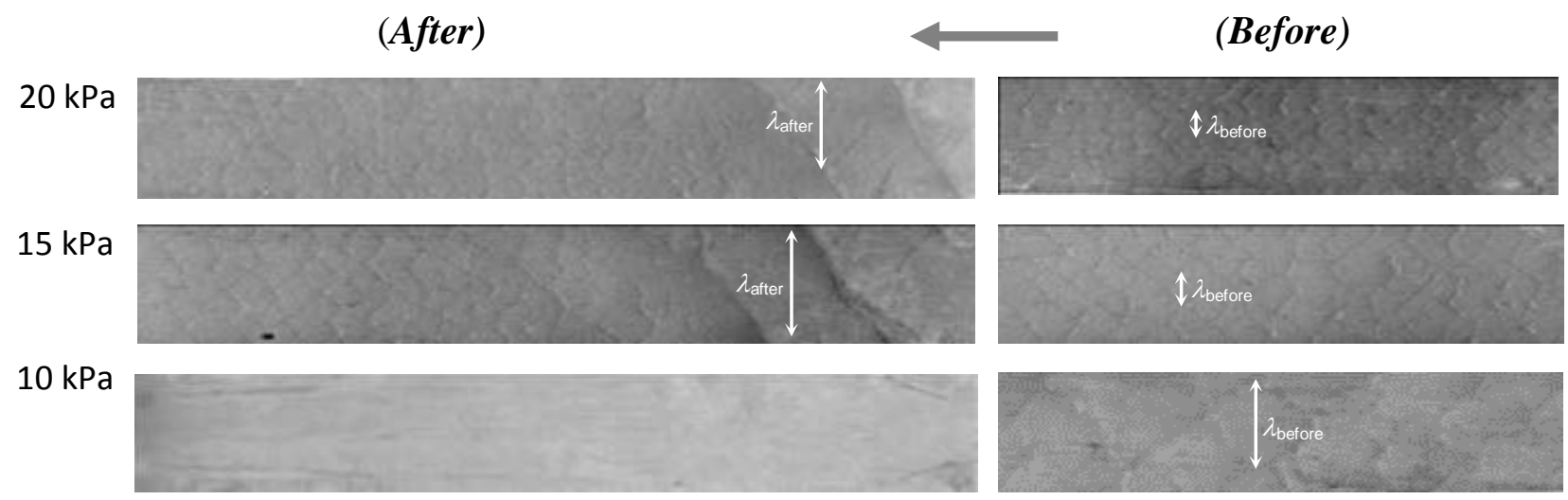

Fig. 4 


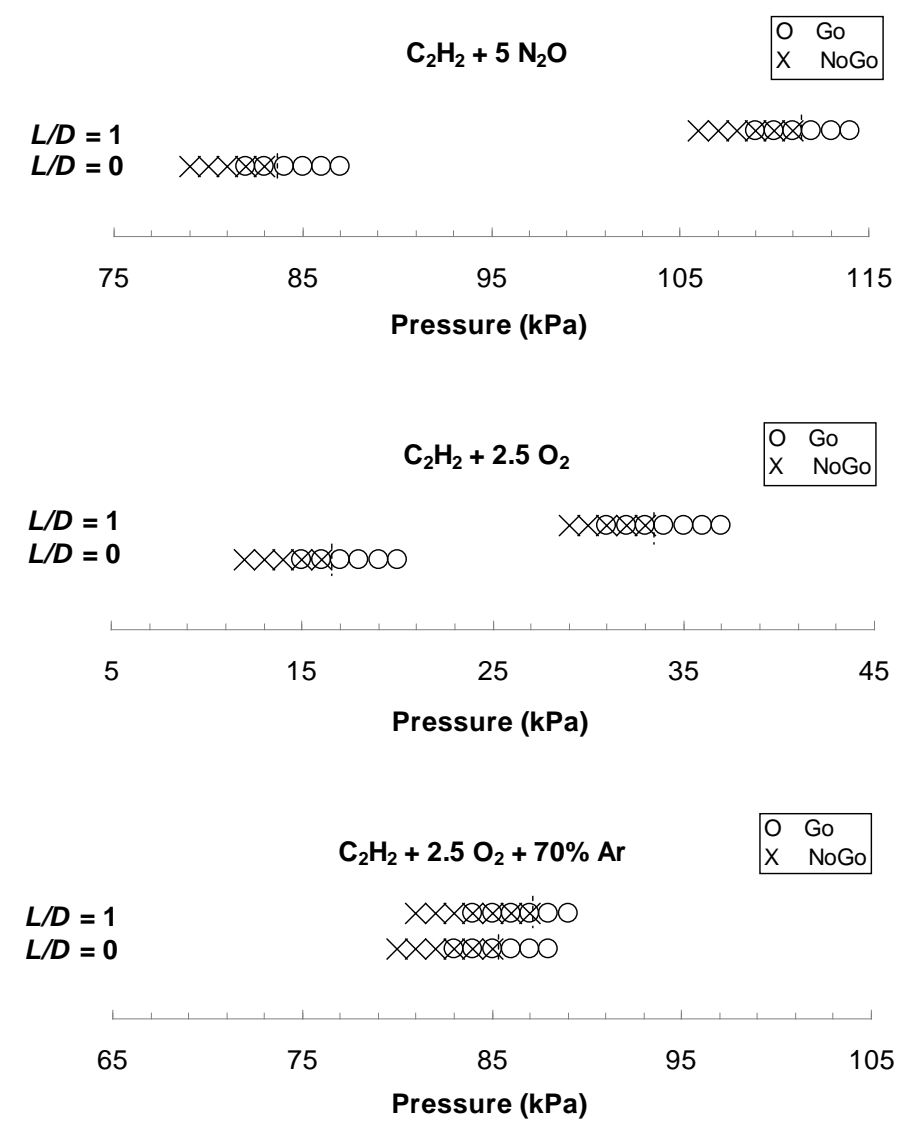

Fig. 5 


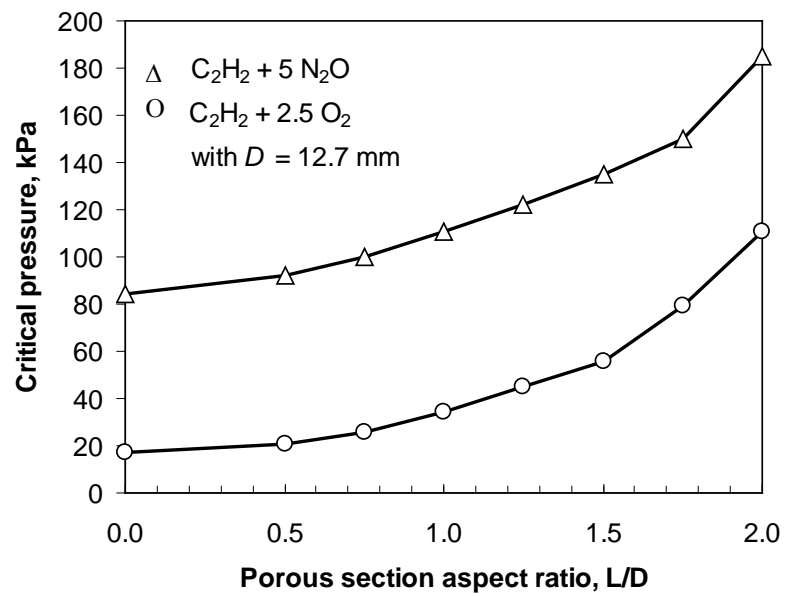

(a)

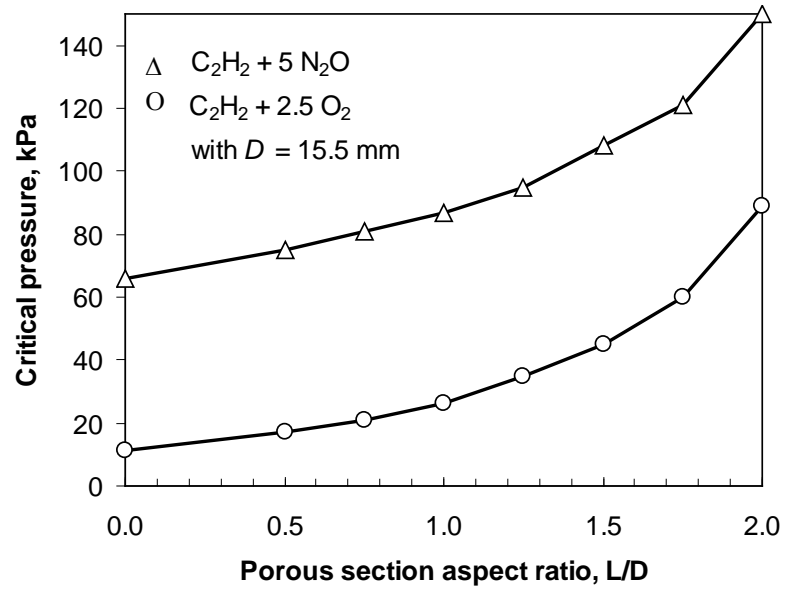

(b)

Fig. 6 


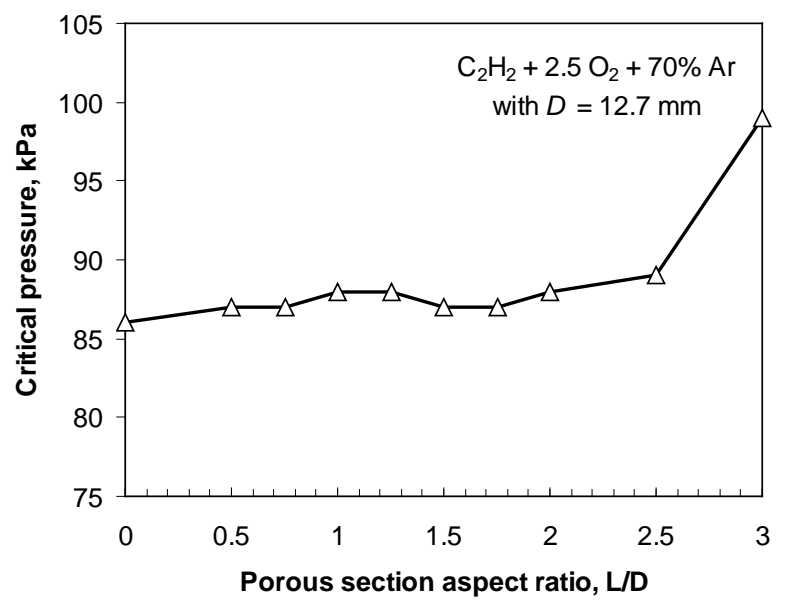

(a)

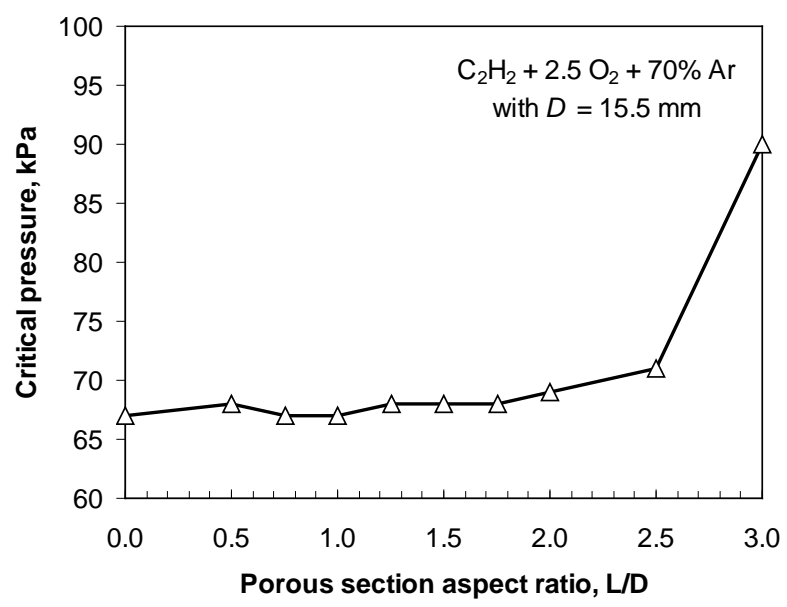

(b)

Fig. 7 